\title{
Spinal Cord Independence Measure: Comprehensive ability rating scale for the spinal cord lesion patient
}

\author{
Amiram Catz, MD, PhD; ${ }^{*}$ Malka Itzkovich, MA, OT \\ Loewenstein Rehabilitation Hospital, Raanana, Israel; Sackler Faculty of Medicine, Tel Aviv University, Tel Aviv, \\ Israel
}

\begin{abstract}
The Spinal Cord Independence Measure (SCIM) is, at present, the only comprehensive rating scale that measures the ability of patients with spinal cord lesions (SCL) to perform everyday tasks according to their value for the patient. This article describes the scale and its scoring techniques, presents the purposes for which SCIM is used, and details its advantages for patients with SCL. Findings of an international multicenter study supported the validity and reliability of its third version, despite intercultural differences, and demonstrated its superior sensitivity to changes in function compared with the Functional Independence Measure. SCIM can be used in patients with SCL for ability assessment, as a compact guide for determining certain treatment goals, and for outcome assessment following interventions designed to promote recovery.
\end{abstract}

Key words: ability rating scales, burden of care, clinical trials, functional assessment, goal determination, Rasch analysis, rehabilitation, reliability, SCIM, scoring techniques, validity.

\section{INTRODUCTION}

Various rating scales have been used to assess ability in persons with spinal cord lesions (SCL), including the Functional Independence Measure (FIM) - the prevailing disability scale-and the Walking Index for Spinal Cord Injury (WISCI) [1]. These scales have been checked for validity to ensure they measure what they were intended to measure [2-3], although scales can be valid and yet measure something other than that in which we are interested.
The validity of FIM has been demonstrated, which means that it measures the burden of care as it was intended to do [2]. But an ability rating scale for patients with SCL is needed to measure not only the burden of care but also functional achievements according to their importance for the patient, which FIM may not be able to measure. For example, a patient may be interested in walking with aids rather than moving in a wheelchair, or in intermittent catheterization rather than in an indwelling catheter, even if these happen to increase the burden of care.

The validity of WISCI has been supported by comparison with other scales that measure mobility [3]. WISCI measures the patient's ability to walk in a physiotherapy gym, from assisted walking between bars to a $10 \mathrm{~m}$ independent walk [3]. But WISCI does not measure other mobility tasks that are relevant to rehabilitation after SCL. Although in a recent study, scores for the second version of WISCI (WISCI-II) showed extremely high correlation (Spearman $r=0.97$ ) with the indoor mobility measured by the Spinal Cord Independence Measure

Abbreviations: ASIA = American Spinal Injury Association, FIM = Functional Independence Measure, $\mathrm{SCI}=$ spinal cord injury, SCIM = Spinal Cord Independence Measure, SCL = spinal cord lesions, WISCI = Walking Index for SCI.

*Address all correspondence to Amiram Catz, MD, PhD, Medical Director; Department IV, Spinal Rehabilitation, Loewenstein Rehabilitation Hospital, 278 Ahuza St, PO Box 3, Raanana 43100, Israel; +972-9-770-9090; fax: +9729-765-4897. Email: amcatz@post.tau.ac.il

DOI: 10.1682/JRRD.2005.07.0123 
(SCIM) [3], the two scales, which were designed for different purposes, measure different abilities. The high correlation is not surprising because the indoor mobility SCIM subscale and WISCI, which was published 3 years after SCIM, refer similarly to devices, braces, and assistance. But WISCI assesses in greater detail one aspect of mobility (10 m walk in a gymnasium), ignoring other aspects assessed by SCIM, such as bed and wheelchair mobility or walking distances outdoors.

The purpose of the measurement affects the selection of a rating instrument. When the purpose is measuring performance of daily activities in patients with SCL, one should consider SCIM.

This article describes SCIM and its scoring techniques, presents the purposes for which the scale is used, and details its advantages for patients with SCL.

\section{DEVELOPMENT OF SCIM}

SCIM is the only comprehensive functional rating scale designed specifically for patients with SCL. Development of SCIM started in 1994. Its first version was presented at the Atlanta American Spinal Injury Association (ASIA) International Society of Paraplegia meeting in 1996 and published in 1997 [4]. Clinical experience and studies have led to a refined second version (SCIM II) published in 2001 [5]. These versions were found sensitive to functional changes in patients with SCL $[4,6]$, reliable and valid, and user-friendly [4-7]. After further studies and consultation with specialists from various countries, a third international version (SCIM III) intended to overcome intercultural differences was formulated in 2002 [8-9].

\section{SCIM FOR MEASURING STATUS OR IMPROVEMENT OF EVERYDAY FUNCTIONS}

At present, SCIM is the only scale that measures independence in all aspects of primary daily activities relevant for patients with SCL, scores independence in performing various tasks according to their value for the patient, and scores every task or area of function according to its relative weight in the total relevant daily function [4-7]. The relative weights of SCIM components were based on a combination of the items' value for patients, predicted difficulty, and time required for per- formance. The value for the patient and the relative weights of the tasks or areas of function were initially determined based on a consensus of Israeli professionals and modified in SCIM III following consultations with European and American experts and in light of Rasch analysis findings [7].

SCIM is easy to use; it requires no manual (scoring criteria are detailed on the evaluation sheet), and the range of the total score is 0 to 100 [4-7]. Because of its SCL patientoriented and user-friendly design, SCIM can be useful for professionals interested in measuring the status or improvement of everyday functions relevant for patients with SCL.

\section{SCIM FOR TREATMENT GOAL DETERMINATION}

SCIM can also be used as a compact guide for determining treatment goals. In addition to assessing ability, SCIM defines quantitative goals for functional restoration of primary daily activities. The SCIM form [7] can help the professionals who care for the patient determine which of the primary daily tasks should be improved and how the performance of that task should change so the patient can achieve a higher ability.

\section{SCIM FOR OUTCOME ASSESSMENT IN CLINICAL TRIALS}

SCIM is used not only for clinical purposes but also for quantitative functional outcome assessment following interventions designed to promote recovery from spinal cord injury (SCI) and to increase functional achievements [10-11]. The patients' objectives are not limited to prevention of secondary degeneration; electrophysiological improvement; histological evidence for regeneration; and ability to move limbs, feel, or sweat. Patient objectives also include breathing ability, sphincter control, and independent mobility and self-care.

Moreover, by defining the goals of primary daily activity restoration, SCIM can help standardize the rehabilitation of these activities following intervention for SCI recovery and thereby prevent a considerable part of rehabilitation from becoming a confounding factor in clinical trials. 


\section{SCORING PERFORMANCE WITH SCIM}

SCIM III covers 19 tasks, all activities of daily living, grouped into four areas of function (subscales): Self-Care (scored 0-20), Respiration and Sphincter Management (0-40), Mobility in Room and Toilet (0-10), Mobility Indoors and Outdoors (0-30) (Appendix, available online only at http://www.rehab.research.va.gov/). The domains of each subscale were determined by a consensus of experienced professionals to enable unidimensionality of SCIM subdivisions, which was verified by Rasch and factor analyses [7]. SCIM III incorporates changes to SCIM II, as detailed in Table 3 of the article on the Rasch analysis of SCIM II [7]. However, items that according to the analysis may overlap were not deleted to ensure that daily tasks important to some patients were not overlooked. Additional changes were implemented following comments by raters and expert advice.

Ideally, SCIM is scored by observation. Each subscale is best scored by a team member experienced in assessment and treatment in the domain covered by the subscale. But SCIM can also be scored by a single team member or by interview (which slightly decreases its scoring precision) [12-13].

SCIM items are scored by observing the patient performing a task and choosing the score most compatible with the patient's performance from those listed in the SCIM evaluation sheet next to the scoring criterion definition. For tasks such as sphincter management, which may not be observable by the scorer, the rater is advised to consult the patient's records or a professional team member who observed the patient performing the task.

For many SCIM items, performance is observed similarly to WISCI and FIM. However, SCIM scores some items differently from WISCI and FIM, and some items scored by SCIM are not addressed by WISCI or FIM. Among these items are feeding (not eating), respiration, bladder management, mobility in bed and action to prevent pressure sores, mobility for moderate distances (10-100 m), wheelchair-car transfer, and ground-wheelchair transfer.

Clips and animations of patients performing these tasks were presented at the ASIA meeting precourse on outcome measures held in May 2005 in Dallas, Texas. The audience enthusiastically practiced scoring the patients with SCIM, using SCIM forms and the interactive system. Most participants had no difficulty scoring the items correctly, although some members of the audience were not absolutely clear about some items or disagreed with some of the authors' suggestions for scoring.
A manual may have improved the clarity of the items and the reliability of SCIM, but SCIM reliability was found reasonable [5-7], even without a manual, which may even impose more of a burden on raters.

\section{FINDINGS OF INTERNATIONAL SCIM STUDY}

SCIM III has recently undergone an international multicenter evaluation, with 425 participants from Canada, Germany, Denmark, England, Italy, and Israel. Findings showed total agreement between raters was 74.5 to 96.2 percent for all SCIM tasks, with $\kappa$ values between 0.631 and $0.823(p<0.001)$ and intraclass correlation coefficient values from 0.94 to 0.97 . Cronbach $\alpha$ was above 0.7 [8].

Most SCIM III items had an acceptable fit to the Rasch model (mean square fit values of 0.6-1.4), which implies that categories intended to represent higher difficulty are passed by fewer people and by people with higher abilities. According to the Rasch analysis, the raters scored patient ability and not other patient properties, used item categories in the correct and hierarchical order, and differentiated well between levels of patient ability and item difficulty. Item hierarchy was found stable across most clinical subgroups and across countries [9].

The coefficient of Pearson's correlation between FIM and SCIM III was $0.79(p<0.01)$ [9]. The McNemar test showed that SCIM was significantly more sensitive than FIM to changes in total score between the first and second examination (changes $>1$ raw score point, the least detectable) in Respiration and Sphincter Management, as well as in the Mobility Indoors and Outdoors subscales $(p<0.001)$. In the other two subscales, Self-care and Mobility in Room and Toilet, the differences between the two scales were nonsignificant [8].

The findings supported the validity and reliability of SCIM III despite intercultural differences, demonstrated its superior sensitivity to changes in function compared with FIM, and justified the use of SCIM in clinical research, including cross-cultural trials.

\section{CONCLUSIONS}

SCIM is the only comprehensive ability rating scale designed specifically for patients with SCL. It can be used for assessment of the ability to perform everyday tasks according to their value for the patient with SCL, as 
a compact guide for treatment goal determination, and for outcome assessment following interventions designed to promote recovery from SCI.

Despite its advantages, the SCIM III version is not perfect. At the Dallas ASIA annual meeting precourse on outcome measures, Dr. Marca Sipski welcomed spinal units to contribute to its further development, study, and improvement.

\section{ACKNOWLEDGMENTS}

This material was unfunded at the time of manuscript preparation.

The authors are the developers of the SCIM.

\section{REFERENCES}

1. Catz A, Itzkovich M. Trends in the assessment of functional outcomes after spinal cord lesions. In: Soroker N, Ring $\mathrm{H}$, editors. Advances in physical and rehabilitation medicine. Bologna (Italy): Monduzzi; 2003. p. 123-27.

2. Dodds TA, Martin DP, Stolov WC, Deyo RA. A validation of the functional independence measurement and its performance among rehabilitation inpatients. Arch Phys Med Rehabil. 1993;74(5):531-36. [PMID: 8489365]

3. Morganti B, Scivoletto G, Ditunno P, Ditunno JF, Molinari M. Walking Index for Spinal Cord Injury (WISCI): Criterion validation. Spinal Cord. 2005;43(1):27-33. [PMID: 15520841]

4. Catz A, Itzkovich M, Agranov E, Ring H, Tamir A. SCIM-Spinal Cord Independence Measure: A new disability scale for patients with spinal cord lesions. Spinal Cord. 1997;35(12):850-56. [PMID: 9429264]

5. Catz A, Itzkovich M, Steinberg F, Philo O, Ring H, Ronen J, Spasser R, Gepstein R, Tamir A. The Catz-Itzkovich SCIM: A revised version of the Spinal Cord Independence Measure. Disabil Rehabil. 2001;23(6):263-68. [PMID: 11336099]

6. Catz A, Itzkovich M, Tamir A, Philo O, Steinberg F, Ring H, Ronen J, Spasser R, Gepstein R. SCIM-Spinal cord independence measure (version II): sensitivity to functional changes [Hebrew]. Harefuah. 2002;141(12):1025-31. [PMID: 12534198]
7. Itzkovich M, Tripolski M, Zeilig G, Ring H, Rosentul N, Ronen J, Spasser R, Gepstein R, Catz A. Rasch analysis of the Catz-Itzkovich spinal cord independence measure. Spinal Cord. 2002;40(8):396-407. [PMID: 12124666]

8. Itzkovich M, Gelernter I, Biering-Sorensen F, Weeks C, Laramee MT, Craven BC, Tonack M, Hitzig SL, Glaser E, Zeilig G, Aito S, Scivoletto G, Mecci M, Chadwick RJ, El Masry WS, Osman A, Glass CA, Silva P, Soni BM, Gardner BP, Savic G, Bergstrom EM, Bluvstein V, Ronen J, Catz A. The Spinal Cord Independence Measure (SCIM) version III: Reliability and validity in a multi-center international study. Disabil Rehabil. In press 2007.

9. Catz A, Itzkovich M, Tesio L, Biering-Sorensen F, Weeks C, Laramee MT, Craven BC, Tonack M, Hitzig SL, Glaser E, Zeilig G, Aito S, Scivoletto G, Mecci M, Chadwick RJ, El Masry WS, Osman A, Glass CA, Silva P, Soni BM, Gardner BP, Savic G, Bergstrom EM, Bluvstein V, Ronen J. A multi-center international study on the Spinal Cord Independence Measure, version II: Rasch psychometric validation. Spinal Cord. 2006. Epub 2006 Aug 15.

10. Grijalva I, Guizar-Sahagun G, Castaneda-Hernandez G, Mino D, Maldonado-Julian H, Vidal-Cantu G, Ibarra A, Serra O, Salgado-Ceballos H, Arenas-Hernandez R. Efficacy and safety of 4-aminopyridine in patients with longterm spinal cord injury: A randomized, double-blind, placebo-controlled trial. Pharmacotherapy. 2003;23(7):823-34. [PMID: 12885095]

11. Popovic MR, Thrasher TA, Adams ME, Takes V, Zivanovic V, Tonack MI. Functional electrical therapy: Retraining grasping in spinal cord injury. Spinal Cord. 2006;44(3): 143-51. [PMID: 16130018]

12. Catz A, Itzkovich M, Steinberg F, Philo O, Ring H, Ronen J, Spasser R, Gepstein R, Tamir A. Disability assessment by a single rater or a team: a comparative study with the CatzItzkovich Spinal Cord Independence Measure. J Rehabil Med. 2002;34(5):226-30. [PMID: 12392238]

13. Itzkovich M, Tamir A, Philo O, Steinberg F, Ronen J, Spasser R, Gepstein R, Ring H, Catz A. Reliability of the Catz-Itzkovich Spinal Cord Independence Measure assessment by interview and comparison with observation. Am J Phys Med Rehabil. 2003;82(4):267-72. [PMID: 12649651]

Submitted for publication July 10, 2005. Accepted in revised form December 13, 2005. 